\title{
The influence of alien translocations on in vitro androgenesis in lines of spring common wheat
}

Timonova E.M. ${ }^{1,2 *}$, Adonina I.G. ${ }^{1,2}$, Salina E.A. ${ }^{2}$

${ }^{1}$ Institute of Cytology and Genetics, SB RAS, Novosibirsk, Russia

${ }^{2}$ Kurchatov Genomic Center of the Institute of Cytology and Genetics, SB RAS, Novosibirsk, Russia

*email:eegorova@bionet.nsc.ru

Androgenesis is potentially the most effective technique for doubled haploid production of wheat. However, it is not widely used in breeding programs due to its main limitation: the genotype dependence. The basic approach to the production of new genotypes of wheat is introgressive hybridization and enrichment of the Triticum aestivum L. gene pool by genes from different species of goat grass, wheatgrass, rye and other relatives. Due to genetic differences between wheat and related species, it was assumed that alien genetic material is different in their capacity to conduct androgenesis. The aim of this work was to develop a set of doubled haploid (DH) wheat lines containing alien translocation and to study their influence on the ability to androgenesis in anther culture of those lines. In the experiment we used: cultivar Novosibirskaya 16, line 991 carrying translocations 1RS.1BL from rye and 5BS.5BL-5SL from Aegilops speltoides and four hybrid $F_{3}$ generation lines $(10-7,14-8,15-8,15-12)$ from their crossing and differing in the content of foreign translocations. A total of 4770 anthers were tested. It was shown that parameters of androgenesis such as the number of embryo-like structures, the total number of regenerants, and the number of green regenerants per 100 anthers varied depending on line. The best-responding lines $991,10-7$ and 14-8 are characterized by the presence of $1 \mathrm{RS} .1 \mathrm{BL}$ wheat-rye translocation chromosome. Regeneration frequency of green plants was recorded to be 4,0,4,2 and 11,3\% respectively. It could be concluded that short arm of the chromosome 1R carries genes that stimulates androgenesis in anther culture. Frequency of albino plants for the cultivar Novosibirskaya 16 was high $(7,2 \%)$ opposite to frequency of green plants $(0,2 \%)$. Spontaneous doubled haploids, verified by cytological analysis of chromosome number and by the measurement of stomata guard cell length, were found in $39,1 \%$ of plants.

Acknowledgements: This work was done within the framework of State Assignment Kurchatov Genomic Center of the Institute of Cytology and Genetics, SB RAS (075-152019-1662). 